\title{
Posttraumatic Cerebral Infarction After Decompressive Craniectomy for Traumatic Brain Injury: Incidence, Risk Factors and Outcome
}

\author{
Tsung-Ming SU', Chu-Mei LAN², Tsung-Han LEE ${ }^{1}$, Fu-Yuan SHIH ${ }^{1}$, Shih-Wei HSU³ , Cheng-Hsien LU ${ }^{4}$ \\ ${ }^{1}$ Kaohsiung Chang Gung Memorial Hospital and Chang Gung University, College of Medicine, Department of Neurosurgery, Kaohsiung, Taiwan \\ ${ }^{2}$ Chang Jung Christian University, Department of Health Psychology, Tainan, Taiwan \\ ${ }^{3}$ Kaohsiung Chang Gung Memorial Hospital and Chang Gung University, College of Medicine, Department of Radiology, Kaohsiung, Taiwan \\ ${ }^{4}$ Kaohsiung Chang Gung Memorial Hospital and Chang Gung University, College of Medicine, Department of Neurology, Kaohsiung, Taiwan
}

\section{ABSTRACT}

AIM: To investigate the incidence, timing, risk factors of posttraumatic cerebral infarction (PTCl) and its influence on mortality in patients with moderate to severe traumatic brain injury (TBI).

MATERIAL and METHODS: After reviewing the medical records and radiographs over a 6-year period, 173 patients with moderate to severe TBI were enrolled to determine the risk factors for the development of PTCI following unilateral decompressive craniectomy (DC).

RESULTS: The incidence of PTCI following DC was 31.2\%. Infarction in the posterior cerebral artery territory was the most common site of PTCI. The PTCl group had a significantly increased mortality $(p<0.001)$ and unfavorable outcome $(p<0.001)$. After stepwise logistic regression analysis, preoperative Glasgow Coma Scale (GCS) score $(p<0.001$, odds ratio [OR] $=0.536,95 \%$ confidence interval $[\mathrm{Cl}]=0.407-0.706)$, pupillary dilation $(\mathrm{p}=0.016, \mathrm{OR}=3.2,95 \% \mathrm{Cl}=1.24-8.28)$, subdural hematoma $(\mathrm{p}=0.01, \mathrm{OR}=16.87,95 \%$ $\mathrm{Cl}=1.97-144.30)$ and craniectomy size $(\mathrm{p}=0.017, \mathrm{OR}=1.02,95 \% \mathrm{Cl}=1.0-1.04)$ remained independently associated with $\mathrm{PTCl}$ development following DC.

CONCLUSION: PTCI is a severe complication in patients with acute TBI. We recommend repeating computed tomography within 3 days of trauma to detect the occurrence of PTCI in patients with subdural hematoma who have low preoperative GCS score and pupillary dilation, irrespective of neurological status. More studies are necessary to clarify the role and benefit of DC in patients with a GCS score of 5 or less.

KEYWORDS: Decompressive craniectomy, Posttraumatic cerebral infarction, Traumatic brain injury

ABBREVIATIONS: ACA: Anterior cerebral artery, Cl: Confidence interval, CT: Computed tomography, DC: Decompressive craniectomy, GCS: Glasgow Coma Scale, GOS: Glasgow Outcome Scale, ICP: Intracranial pressure, MCA: Middle cerebral artery, OR: Odds ratio, PCA: Posterior cerebral artery, PTCI: Posttraumatic cerebral infarction, ROC: Receiver operating characteristic, SAH: Subarachnoid hemorrhage, SDH: Subdural hematoma, TBI: Traumatic brain injury, VB: Vertebrobasilar 


\section{INTRODUCTION}

$\mathrm{D}$ ecompressive craniectomy (DC) has been performed increasingly over the past years to control medically refractory intracranial hypertension in patients with traumatic brain injury (TBI) $(3,12,15)$. Although DC is a potentially life-saving procedure for patients with TBI, it is associated with some potential complications $(1,6,16,17)$. Posttraumatic cerebral infarction (PTCl) is a well-recognized complication of TBI and has been thought as an indicator of poor clinical outcome $(5,11,14,19,20)$. A variety of mechanisms may account for this complication, including systemic hypoperfusion, cerebral vasospasm, direct vascular injury, cerebral thromboembolism, vascular impingement caused by cerebral herniation and venous congestion at the craniectomy site $(9,11,13,14,19,20)$. Early diagnosis of PTCl is often difficult because of the diverse clinical manifestations, the delay in presentation of symptoms, and other associated brain injuries (20). Although there have been few studies defining early predictors of development of PTCI $(11,14,19,20)$, its incidence, timing, risk factors and influence on mortality are not well studied. Early identification of patients who are at particular risk for PTCl would be helpful in managing patients with TBI.

Herein, we retrospectively reviewed a series of patients who underwent unilateral DC to treat moderate or severe TBI over a 6-year period. We examined the incidence and timing of PTCl, and sought to find independent risk factors for the development of PTCl and the influence of PTCl on patient mortality. Our research aimed to improve the diagnosis and therapeutic strategy of this potentially devastating complication in patients undergoing unilateral DC for acute TBI.

\section{MATERIAL and METHODS}

\section{Study Design}

In this study, we retrospectively reviewed the medical records on 287 patients with moderate or severe TBI who had Glasgow Coma Scale (GCS) score of less than 13 on admission and underwent DC from 2007 to 2012. The indication for performing DC was that the brain was markedly swollen after evacuation of any type of intradural hemorrhagic lesion or increased intracranial pressure (ICP) refractory to medical treatment in patients with ICP monitoring. The DC procedure entailed removal of a large portion of skull bone. The underlying dura was opened widely for outward expansion of the swollen brain. Augmentation duraplasty with artificial dural substitute was performed in some patients. In other patients, the dura was left open and the exposed brain surface was covered with Gelfoam sponge (Pfizer, New York, NY, USA).

Among them, 38 patients who had incomplete medical records or lacked postoperative computed tomography (CT) scan were excluded from study. To avoid confounding factors, patients who had no obvious midline shift due to diffuse brain swelling or bilateral hematomas, penetrating brain injury or a history of cerebral infarction prior to their injury were excluded. Finally, 173 patients with moderate or severe TBI were enrolled to determine the risk factors for the development of PTCI following unilateral DC.

\section{Clinical Data}

For each patient, the demographic data, history of medical disease, mechanism of head injury, neurological condition at the time of admission and prior to DC, occurrence of hypotension (systolic blood pressure less than $90 \mathrm{mmHg}$ or/and diastolic pressure less than $40 \mathrm{mmHg}$ for more than 10 minutes), interval between the time of accident and DC, interval between the time of accident and recognition of cerebral infarction by brain CT scan, and functional outcome were reviewed from the medical records. The findings of neurological examination including the preoperative GCS score, the pupillary size and reactivity, and limb movements were carefully recorded. Severe TBI was defined as a GCS score of 8 or less following resuscitation. Moderate TBI was defined as a GCS score of 9 to 12. Decompression time was defined as the interval between the time of accident and the start of DC operation plus 30 minutes, which represented an estimated time of DC procedure.

Functional outcome was evaluated 3 months after patient discharge by Glasgow Outcome Scale (GOS) score as follows: 1 = death, 2 = persistent vegetative state, 3 = severe disability, $4=$ moderate disability and $5=$ good recovery (7). Outcome was categorized into favorable (GOS score 4 and 5 ) and unfavorable (GOS score 1-3) outcomes.

\section{Radiographic Data}

Review of preoperative brain CT scans was performed by one neuroradiologist and two neurosurgeons without knowledge of whether the patient developed PTCI. Low attenuated lesions in well-defined arterial vascular distribution on any brain CT scan within 2 weeks after the accident were defined as PTCI. A diagnosis of cerebral infarction was revised if follow-up studies indicated the findings were actually related to evolving contusions, artifact, or were inconsistently visualized. Sites of infarction territory were recorded according to the following vascular distributions: anterior cerebral artery (ACA) territory, middle cerebral artery (MCA) territory, posterior cerebral artery (PCA) territory, vertebrobasilar (VB) territory and lenticulostriate-thalamoperforating territory.

In addition, presence of intradural hemorrhagic lesions, degree of midline shift, status of basal cistern, presence of calvarial skull fracture and severity of traumatic subarachnoid hemorrhage $(\mathrm{SAH})$ were also recorded from the preoperative CT scans. Intradural hemorrhagic lesions which prompted a DC procedure were separated into two groups for analysis: Cerebral contusion (maximal diameter $\geq 5 \mathrm{~cm}$ ) or subdural hematoma (SDH) with/without contusion. The midline shift on the CT scan was defined as the distance $(\mathrm{mm})$ that septum pellucidum of the brain was displaced away from the midline. Status of basal cistern was classified as normal, compressed or absent (8). The calvarial skull was categorized into five bone plates as the frontal bone, parietal bone, calvarial temporal bone, calvarial sphenoid bone and calvarial occipital bone. Skull fractures involving these five bone plates were included for analysis. However, skull base and facial bone fractures were excluded from analysis. For each patient, the number of fracture-involved bone plate was carefully reviewed. 
The severity of traumatic SAH was determined using a Fisher classification with modification (4). Grade 1 indicated no evidence of SAH. Grade 2 represented $\mathrm{SAH}$ in superior brain convexity with cisternal hemorrhage less than $1 \mathrm{~mm}$. Grade 3 was cisternal hemorrhage more than $1 \mathrm{~mm}$. Grade 4 included only patients with intraventricular hemorrhage. The size of craniectomy was calculated from first postoperative CT scan by assuming the craniectomy area as a hypothetical circle with diameter $=\mathrm{d}$ (the largest diameter calculated on CT scans) $(2,12)$. The area $(A)$ of the segment of a sphere above the craniectomy area was therefore calculated with the formula:

$$
A=\pi\left[(d / 2)^{2}+h^{2}\right]
$$

where $h$ represents the perpendicular line to $d$ with the longest distance from $d$ to the dural flap (Figure 1).

\section{Statistical Analyses}

Two separate statistical analyses were performed. First, the clinical data, including gender, preoperative clinical manifestations and CT findings between the two patient groups, were analyzed by means of the Chi-square test or Fisher's exact test. The mean age, decompressive time, and craniectomy size between the two patient groups were logarithmically transformed to improve normality, and comparisons between the two patient groups were made using the Student's t-test. Second, stepwise logistic regression was then used to evaluate the relationships between the preoperative clinical factors and the presence of PTCl during hospitalization, with adjustments made for other potential confounding factors. The receiver operating characteristic (ROC) curves were generated for evaluating the significant predictor variables of $\mathrm{PTCl}$. All statistical analyses were conducted using the SAS software package, version 9.1 (2002, SAS Statistical Institute, Cary, North Carolina, USA).

\section{RESULTS}

After reviewing the medical records and radiographs, 173 patients with moderate or severe TBI were enrolled to determine the risk factors for the development of PTCI following unilateral DC. Among these patients, 140 patients (80.9\%) sustained severe TBI. Overall, 54 patients (31.2\%) developed PTCI following DC. The other 119 patients did not develop PTCI following DC.

\section{Clinical Data}

Table I shows the demographic data, mechanism of injury, clinical characteristics and radiographic findings. Among 54 patients with $\mathrm{PTCl}$ following DC, the patient subgroup was predominantly composed of male (42 of 54 patients). All but one patient sustained severe TBI. Pupillary dilation before DC was noted in 41 patients $(75.6 \%)$ with $\mathrm{PTCl}$. Comparatively, severe TBI was noted in 87 patients without PTCI. Only 28 patients $(23.5 \%)$ without PTCl developed pupillary dilation before DC. The craniectomy size was significantly larger in patients with PTCl $(\mathrm{p}=0.007)$. Duraplasty was performed in 21 patients (38.9\%) with $\mathrm{PTCl}$ and 47 patients (39.5\%) without PTCl.
In the group of 54 patients with $\mathrm{PTCl}, 26$ patients (48.1\%) died, and 16 patients remained in a persistent vegetative state. Functional outcome was favorable in nine of 54 (16.7\%), and unfavorable in 45 of 54 (83.3\%) patients. Comparatively, the outcome in patients without PTCl was favorable in 85 of $119(71.4 \%)$, and unfavorable in 34 of $119(28.6 \%)$ patients. Only six patients $(5 \%)$ expired after aggressive treatment. The PTCl group had significantly increased mortality $(p<0.001)$ and an unfavorable outcome $(p<0.001)$. The mortality rates were significantly higher in patients with PTCI than without when matched for preoperative GCS score (Table II).

\section{Radiographic Data}

Acute SDH was the most common hemorrhagic lesion for performing DC. Basal cisterns were compressed in nine of 54 and completely obliterated in 45 of 54 patients with PTCI. In patients without $\mathrm{PTCl}$, status of basal cistern was compressed in 68 and absent in 51 patients. Mean midline shift was 12.73 $\mathrm{mm}$ in patients with $\mathrm{PTCl}$, and $9.2 \mathrm{~mm}$ in patients without PTCl $(p<0.001)$

Cerebral infarction developed early in the clinical course, and in 38 patients $(70.3 \%)$, the infarction developed within 3 days after the injury. Mean interval between the time of accident and infarction diagnosis was 54.8 hours. The most common site of PTCl was the PCA territory (Figure 2A, B), but infarcts were also relatively common in the ACA (51.8\%) and MCA (55.6\%) territories (Table III). Other areas of infarction included the lenticulostriate-thalamoperforating territory in 11 patients and VB territory in ten patients. In 36 patients, the infarctions were in more than one vascular territory. All patients with infarction involving VB territory died even with aggressive management. The mortality rate was the lowest in patients

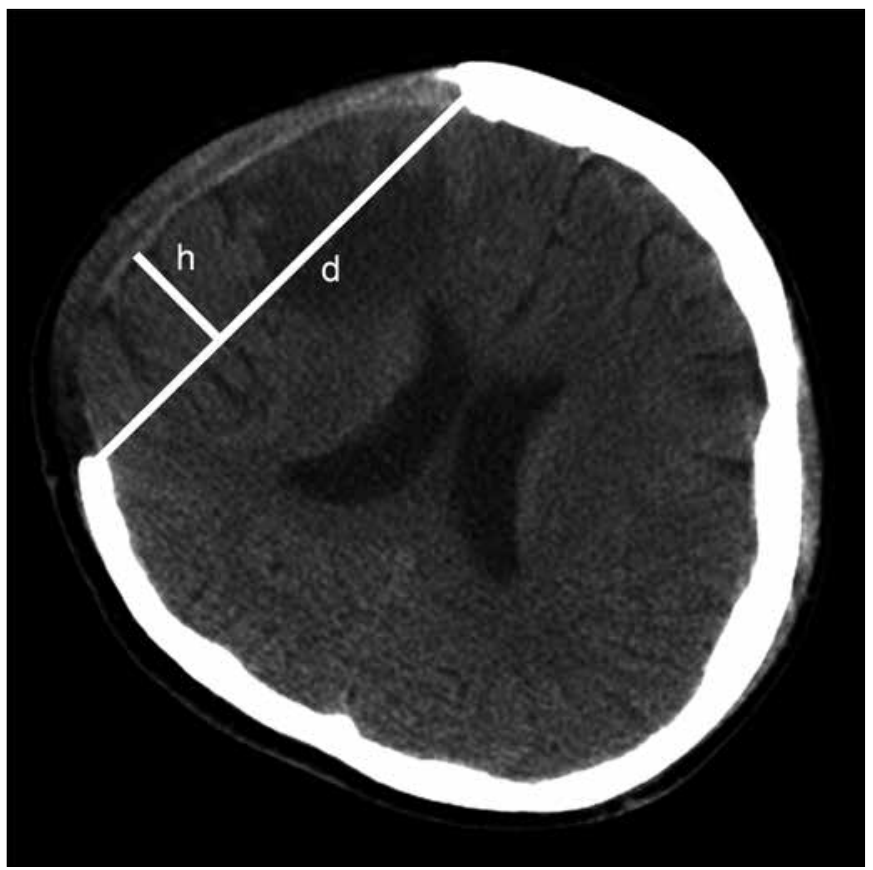

Figure 1: Craniectomy size. Axial computed tomography scan demonstrates the method used for calculation of the craniectomy size (see text for details). 
Table I: Risk Factors for the Development of Posttraumatic Cerebral Infarction*

\begin{tabular}{|c|c|c|c|c|c|}
\hline & $\begin{array}{l}\text { Without PTCI } \\
(n=119)\end{array}$ & $\begin{array}{c}\text { With PTCI } \\
(n=54)\end{array}$ & OR & $95 \% \mathrm{Cl}$ & p value ${ }^{\dagger}$ \\
\hline Sex (male/female) & $84 / 35$ & $42 / 12$ & 1.46 & $0.69-3.10$ & 0.33 \\
\hline Age (year) & $50.0 \pm 18.8$ & $51.6 \pm 18.1$ & & & 0.13 \\
\hline \multicolumn{6}{|l|}{ Mechanism } \\
\hline Traffic accident & 92 & 39 & & & 0.161 \\
\hline Falling & 25 & 11 & & & \\
\hline Others & 2 & 4 & & & \\
\hline \multicolumn{6}{|l|}{ Underlying diseases } \\
\hline Hypertension & 24 & 12 & 1.13 & $0.52-2.47$ & 0.76 \\
\hline Diabetes mellitus & 14 & 7 & 1.12 & $0.42-2.95$ & 0.82 \\
\hline End stage renal disease & 1 & 3 & 6.94 & $0.71-68.33$ & 0.09 \\
\hline \multicolumn{6}{|l|}{ Clinical findings before DC } \\
\hline GCS score & $7.4 \pm 2.1$ & $5.1 \pm 1.4$ & & & $<0.001$ \\
\hline Pupillary dilation & 32 & 41 & 8.58 & 4.08-18.04 & $<0.001$ \\
\hline Hypovolemic shock & 0 & 2 & 0.30 & $0.24-0.38$ & 0.10 \\
\hline \multicolumn{6}{|l|}{ Preoperative CT findings ${ }^{\ddagger}$} \\
\hline \multicolumn{6}{|l|}{ Intradural hemorrhagic lesion } \\
\hline SDH with/without contusion & 91 & 53 & 16.31 & $2.16-123.33$ & $<0.001$ \\
\hline Cerebral contusion & 28 & 1 & & & \\
\hline \multicolumn{6}{|l|}{ Status of basal cistern } \\
\hline Absent & 51 & 45 & 0.15 & $0.067-0.34$ & $<0.001$ \\
\hline Compressed & 68 & 9 & & & \\
\hline Midline shift (mm) & $9.2 \pm 4.2$ & $12.73 \pm 4.9$ & & & $<0.001$ \\
\hline \multicolumn{6}{|l|}{ Fisher grade of traumatic SAH } \\
\hline 4 & 3 & 2 & & & 0.53 \\
\hline 3 & 21 & 10 & & & \\
\hline 2 & 51 & 28 & & & \\
\hline 1 & 44 & 14 & & & \\
\hline \multicolumn{6}{|c|}{ Number of fracture-involved bone plate } \\
\hline 2 & 25 & 14 & & & 0.46 \\
\hline 1 & 42 & 14 & & & \\
\hline 0 & 52 & 26 & & & \\
\hline Decompression time (hour) & $12.2 \pm 7.9$ & $6.0 \pm 3.8$ & & & 0.002 \\
\hline \multicolumn{6}{|l|}{ Treatment } \\
\hline Craniectomy size $\left(\mathrm{cm}^{2}\right)$ & $120.6 \pm 24.1$ & $132.4 \pm 31.1$ & & & 0.007 \\
\hline Duraplasty & 47 & 21 & 0.98 & $0.50-1.88$ & 0.94 \\
\hline \multicolumn{6}{|l|}{ Functional outcome } \\
\hline Favorable & 85 & 9 & 12.5 & 5.51-28.35 & $<0.001$ \\
\hline Unfavorable & 34 & 45 & & & \\
\hline Mortality & 6 & 26 & 17.49 & $6.57-46.57$ & $<0.001$ \\
\hline
\end{tabular}

PTCI: Posttraumatic cerebral infarction, OR: Odds ratio, Cl: Confidence interval, DC: Decompressive craniectomy, GCS: Glasgow Coma Scale, CT: Computed tomography, SDH: Subdural hematoma, SAH: Subarachnoid hemorrhage

*Figures are mean $\pm S D$ or number (\%)

tStatistical significance: $p<0.05$

${ }^{\ddagger} \mathrm{CT}$ findings before decompressive craniectomy 
Su TM. et al: Posttraumatic Cerebral Infarction

Table II: Mortality in Patients with PTCI Versus Mortality in Patients without PTCI, Matched for Preoperative GCS Score

\begin{tabular}{ccccc}
\hline \multirow{2}{*}{ GCS score } & Number of patients & \multicolumn{2}{c}{ Mortality rate (\%) } & Without PTCI \\
\cline { 3 - 5 } & 33 & With PTCI & $2 / 32(6.3 \%)$ & 1.0 \\
\hline $9-12$ & 89 & $6 / 18(33.3 \%)$ & $2 / 71(2.8 \%)$ & 0.001 \\
\hline $6-8$ & 51 & $20 / 35(57.1 \%)$ & $2 / 16(12.5 \%)$ & 0.005 \\
\hline 5
\end{tabular}

PTCI: Posttraumatic cerebral infarction, GCS: Glasgow Coma Scale.

"Statistical significance: $p<0.05$.

Table III: Distribution of Posttraumatic Cerebral Infarction $(n=54)$

\begin{tabular}{lcc}
\hline Vascular territory & Number (\%) & Mortality rate (\%) \\
\hline Anterior cerebral artery & $28(51.8 \%)$ & $18(64.3 \%)$ \\
\hline Middle cerebral artery & $30(55.6 \%)$ & $20(66.7 \%)$ \\
\hline Posterior cerebral artery & $45(83.3 \%)$ & $25(55.6 \%)$ \\
\hline Vertebrobasilar territory & $10(18.5 \%)$ & $10(100 \%)$ \\
\hline Lenticulostriate-thalamoperforating territory & $11(20.3 \%)$ & $8(72.7 \%)$ \\
\hline
\end{tabular}
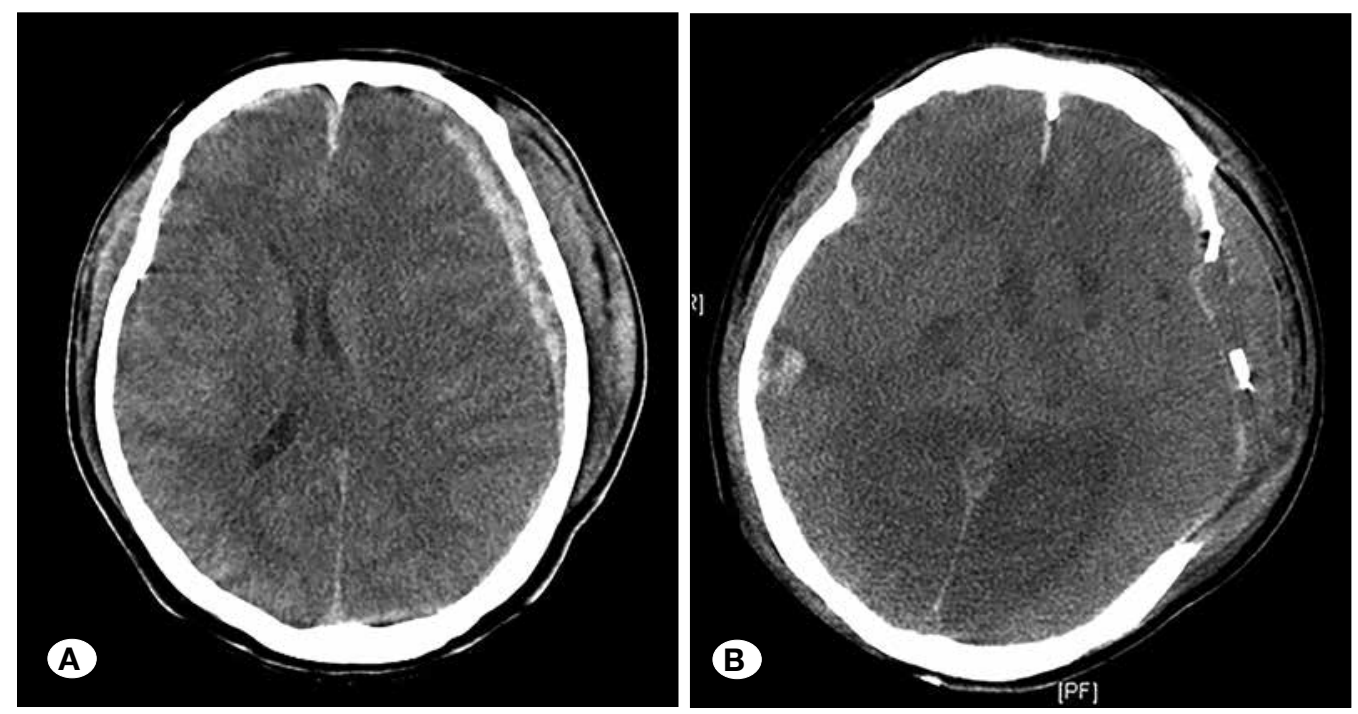

Figure 2: Posttraumatic cerebral infarction. A) The initial axial computed tomography (CT) scan demonstrates a left-sided subdural hematoma with marked midline shift. B) Postoperative axial CT scan reveals multiple infarctions in bilateral posterior cerebral artery and lenticulostriatethalamoperforating territories.

with infarction only involving the PCA distribution. In 12 patients with infarction only in the PCA territory, only two died despite aggressive treatment.

\section{Risk Factors Analyses}

The risk factors associated with the development of $\mathrm{PTCl}$ in patients following TBI were listed in Table I. Statistical analysis of the preoperative clinical manifestations and CT scan findings between the two groups revealed that pupillary dilation $(p<0.001)$, preoperative GCS score $(p<0.001)$, midline shift $(p<0.001), \operatorname{SDH}(p<0.001)$, absent cistern pattern $(p<0.001)$, decompression time $(p=0.002)$ and craniectomy size $(p=0.007)$ were significant variables. Further, these variables were used in the stepwise logistic regression models. After analyses, only preoperative GCS score $(p<0.001$, odds ratio $[\mathrm{OR}]=0.536,95 \%$ confidence interval $[\mathrm{Cl}]=0.407-0.706)$, pupillary dilation $(\mathrm{p}=0.016, \mathrm{OR}=3.2,95 \% \mathrm{Cl}=1.24-8.28), \mathrm{SDH}$ $(\mathrm{p}=0.01, \mathrm{OR}=16.87,95 \% \mathrm{Cl}=1.97-144.30)$ and craniectomy size $(p=0.017, \quad O R=1.02, \quad 95 \% \quad C l=1.0-1.04)$ remained independently associated with $\mathrm{PTCl}$ development and the rate of PTCl development would increase by $41 \%$ with every 1 -point decrease in preoperative GCS score. The area under the ROC curve for preoperative GCS score was $0.816(95 \%$ $\mathrm{Cl}=0.75-0.88, \mathrm{p}<0.001)$ and the cut-off value to predict the development of PTCI following DC for preoperative GCS score was 5.5 (sensitivity $86 \%$ and specificity $65 \%$ ). 


\section{DISCUSSION}

Because of the heterogeneous patient groups in the reported literatures, the incidence of $\mathrm{PTCl}$ in $\mathrm{TBI}$ remains unclear. Mirvis et al. reported that $1.9 \%$ of patients with TBI developed cerebral infarctions (11). In the study of Tawil and colleagues, the incidence of PTCI in patients with severe TBI was $8 \%$ (19). Tian et al. reported that $11.96 \%$ of patients with moderate to severe TBI developed PTCI (20). In our study, the incidence of PTCl was $31.2 \%$ in patients with moderate to severe TBI following unilateral DC. Among our study group, most patients (80.9\%) sustained severe TBI (Table II). TBI patients with a GCS score of 5 or less accounted for $36.4 \%$ of patients with severe $\mathrm{TBI}$. This result might reflect the greater severity of $\mathrm{TBI}$ in our patients. High incidence $(68.6 \%)$ of $\mathrm{PTCl}$ was also noted in patients with a GCS score of 5 or less (Table II). These findings may explain the high incidence of PTCI in our patients. Another reason may be the policy of routine early postoperative CT scan to evaluate intracranial status in our hospital that leads to the diagnosis of PTCl.

Gross mechanical shift of the brain and herniation across the falx and/or tentorium was thought to be the most important cause for PTCl $(11,19,20)$. In our study, preoperative GCS score, pupillary dilation, SDH and craniectomy size were significant risk factors for PTCl development in the stepwise logistic regression models. Low preoperative GCS score and pupillary dilation may indicate that patients suffered a severe TBI. More severe TBI can be expected to produce more severely increased ICP, which results in more severe mass effect to thus favor the development of cerebral infarction. SDH may produce more diffuse mass effect than cerebral contusion and therefore cause cerebral infarction. Besides, midline shift and decompression time were significant risk factors for $\mathrm{PTCl}$ development in univariate analysis. More severe midline shift may reflect more severe mass effect that may lead to the development of cerebral infarction. Similarly, the shorter decompression time may reflect the patient sustained severe TBI that prompted early DC.

Vasospasm associated with traumatic $\mathrm{SAH}$ was thought to be a possible mechanism of PTCI $(10,18)$. However, our study failed to prove that the severity of traumatic SAH was a risk factor for the development of PTCI. In our study, craniectomy size was a significant risk factor for the development of PTCI. DC can allow swollen brain tissue bulging through the craniectomy defect and rapidly relieve the increased ICP. Tian et al. believed that brain tissues and vessels passing through the bony defect were compressed by the dural and bony margin, leading to further congestion, edema and ischemia of the local tissues, which may contribute to the development of PTCI (20). Larger craniectomy size may facilitate these effects and increase the risk of PTCl. Augmentation duraplasty may be beneficial in decreasing the risk of PTCl by alleviating the brain bulging through the bony defect. However, our study failed to demonstrate the influence of duraplasty on the development of PTCl. The higher probability of PTCI after greater DC may also be influenced by the severity of clinical and radiological condition of the patients in whom a greater DC was made. In our opinion, when we face those patients with severe mass effect during surgery, we should not be reluctant to perform adequate DC just because of possible risk for PTCl development.

PTCI has been thought as an indicator of poor clinical outcome $(5,11,14,19,20)$. The development of PTCI may reflect that the $\mathrm{TBI}$ is severe enough to cause PTCl and leads to the poor outcome. In our study, the PTCl group had a significantly unfavorable outcome, as compared with the group without infarction. All patients with infarction involving VB territory died even with aggressive management. The mortality rate was the lowest in patients with infarction only involving the PCA distribution. This result is compatible with the study of Ham and colleagues (5).

The possible reason of high mortality rate $(48.1 \%)$ in our patients with $\mathrm{PTCl}$ is probably erroneous indication for decompressive surgery. Careful selection of patients is crucial in order to avoid unnecessarily aggressive procedures in patients who have little chance of benefit. Our study found the rate of $\mathrm{PTCl}$ development would increase by $41 \%$ with every 1-point decrease in preoperative GCS score. The cutoff value to predict the development of PTCI following DC for preoperative GCS score was 5.5. In our study, 35 of 51 patients with GCS score of 5 or less developed PTCI (Table II). Among these 35 patients, 20 died and 11 remained in a persistent vegetative state. Other 16 patients with a GCS score of 5 or less did not developed PTCl. Among these 16 patients, two died and eight remained in a persistent vegetative state. This finding may support the recommendation of Ham et al. that surgical decompression is probably not indicated in patients with a GCS score of 5 or less because their outcome is usually dismal (5). In our opinion, a conservative approach for DC in patients with a GCS score of 5 or less is still controversial and more studies are necessary to clarify this issue.

The treatment of PTCl lies in prevention. Avoidance of hypotension and hypoxemia is crucial to prevent occurrence of PTCl. The optimal timing for DC is unclear; however, findings from a recent study demonstrated improved outcome with early DC (3). Because DC can allow swollen brain tissue bulging through the craniectomy defect and lead to rapid relief of raised ICP, it is reasonable to perform the procedure early in the posttraumatic period, before irreversible damage has ensured with no chance of recovery. Although the timing of $\mathrm{PTCl}$ development after the trauma remains unclear, we recommend repeating CT examination within 3 days of trauma to detect the occurrence of PTCl, because PTCl was diagnosed within 3 days of trauma in most of our patients. This would help in timely diagnosis of PTCl.

\section{- CONCLUSION}

$\mathrm{PTCl}$ is a severe complication in patients with acute TBI, resulting in poor outcome and high mortality. Preoperative GCS score, pupillary dilation, SDH and craniectomy size are significant risk factors for PTCl development following DC. We recommend repeating CT examination within 3 days of trauma to detect the occurrence of PTCl in patients with SDH who have low preoperative GCS score and pupillary dilation, 
irrespective of postoperative neurological condition of the patient. More studies are necessary to clarify the role and benefit of DC in patients with a GCS score of 5 or less.

\section{- REFERENCES}

1. Ban SP, Son YJ, Yang HJ, Chung YS, Lee SH, Han DH: Analysis of complications following decompressive craniectomy for traumatic brain injury. J Korean Neurosurg Soc 48:244-250, 2010

2. De Bonis P, Pompucci A, Mangiola A, Rigante L, Anile C: Posttraumatic hydrocephalus after decompressive craniectomy: An underestimated risk factor. J Neurotrauma 27:1965-1970, 2010

3. Eberle BM, Schnuriger B, Inaba K, Gruen JP, Demetriades D, Belzberg H: Decompressive craniectomy: Surgical control of traumatic intracranial hypertension may improve outcome. Injury 41:894-898, 2010

4. Fisher CM, Kistler JP, Davis JM: Relation of cerebral vasospasm to subarachnoid hemorrhage visualized by computerized tomographic scanning. Neurosurgery 6:1-9, 1980

5. Ham HY, Lee JK, Jang JW, Seo BR, Kim JH, Choi JW: Posttraumatic cerebral infarction: Outcome after decompressive hemicraniectomy for the treatment of traumatic brain injury. $\mathrm{J}$ Korean Neurosurg Soc 50:370-376, 2011

6. Honeybul S: Complications of decompressive craniectomy for head injury. J Clin Neurosci 17:430-435, 2010

7. Jennett $B$, Bond $M$ : Assessment of outcome after severe brain damage: A practical scale. Lancet 1:480-484, 1975

8. Maas AIR, Hukkelhoven CWPM, Marshall LF, Steyerberg EW: Prediction of outcome in traumatic brain injury with computed tomographic characteristics: A comparison between the computed tomographic classification and combinations of computed tomographic predictors. Neurosurgery 57:11731182, 2005

9. Macpherson P, Graham DI: Arterial spasm and slowing of the cerebral circulation in the ischaemia of head injury. J Neurol Neurosurg Psychiatry 36:1069-1072, 1973
10. Martin NA, Doberstein C, Zane C, Caron MJ, Thomas K, Becker DP: Posttraumatic cerebral arterial spasm: Transcranial Doppler ultrasound, cerebral blood flow, and angiographic findings. J Neurosurg 77:575-583, 1992

11. Mirvis SE, Wolf AL, Numaguchi Y, Corradino G, Joslyn $\mathrm{JN}$ : Posttraumatic cerebral infarction diagnosed by CT: Prevalence, origin, and outcome. AJNR Am J Neuroradiol 11:355-360, 1990

12. Munch E, Horn P, Schurer L, Piepgras A, Paul T, Schmiedek P: Management of severe traumatic brain injury by decompressive craniectomy. Neurosurgery 47:313-323, 2000

13. Rothfus WE, Goldberg AL, Tabas JH, Deeb ZL: Callosomarginal infarction secondary to transfalcial herniation. AJNR Am J Neuroradiol 8:1073-1076, 1987

14. Server A, Dullerud R, Haakonsen M, Nakstad PH, Johnsen UL, Magnaes B: Post-traumatic cerebral infarction. Neuroimaging findings, etiology and outcome. Acta Radiol 42:254-260, 2001

15. Skoglund TS, Eriksson-Ritzen C, Jensen C, Rydenhag B: Aspects on decompressive craniectomy in patients with traumatic head injuries. J Neurotrauma 23:1502-1509, 2006

16. Stiver SI: Complications of decompressive craniectomy for traumatic brain injury. Neurosurg Focus 26:E7, 2009

17. Su TM, Lee TH, Huang YH, Su FW, Chen WF: Contralateral subdural effusion after decompressive craniectomy in patients with severe traumatic brain injury: Clinical features and outcome. J Trauma 71:833-837, 2011

18. Taneda M, Kataoka K, Akai F, Asai T, Sakata I: Traumatic subarachnoid hemorrhage as a predictable indicator of delayed ischemic symptoms. J Neurosurg 84:762-768, 1996

19. Tawil I, Stein DM, Mirvis SE, Scalea TM: Posttraumatic cerebral infarction: Incidence, outcome, and risk factors. J Trauma 64:849-853, 2008

20. Tian HL, Geng Z, Cui YH, Hu J, Xu T, Cao HL, Chen SW, Chen $\mathrm{H}$ : Risk factors for posttraumatic cerebral infarction in patients with moderate or severe head trauma. Neurosurg Rev 31:431437,2008 